\title{
Paclitaxel-coated balloons for the treatment of patients with in-stent restenosis: A meta-analysis of angiographic and clinical data
}

\author{
JUN LI，WAN-LI LIU，XIN YI，GAO-KE FENG，ZHAO LU，XUE-JUN JIANG and XIAO-YAN LI \\ Department of Cardiology, Renmin Hospital of Wuhan University, Wuhan, Hubei 430060, P.R. China
}

Received July 1, 2014; Accepted March 17, 2015

DOI: $10.3892 /$ etm.2015.2431

\begin{abstract}
Paclitaxel-coated balloons (PCBs) have become attractive alternative treatment options for patients with in-stent restenosis (ISR); however, the safety and efficacy of PCBs in comparison with those of conventional therapies are less well defined. The aim of this meta-analysis was to systematically review the efficacy and safety of PCBs for patients with ISR using comparisons with control groups. Electronic databases, such as MEDLINE, Embase and the Cochrane Central Register of Controlled Trials, were searched, and eligible studies that compared PCBs with uncoated balloons (UCBs) or drug-eluting stents (DESs) in patients with ISR were considered. Subgroup analyses were performed with different control groups. Nine studies (1,488 patients, 1,608 lesions) were included in the meta-analysis. Compared with patients who underwent UCB angioplasty, those who underwent PCB angioplasty exhibited a clear superiority in late lumen loss (LLL) [weighted mean difference (WMD), $-0.46 ; 95 \%$ confidence interval (CI), (-0.59)-(-0.34); $\mathrm{P}<0.00001]$ and major adverse cardiac events (MACEs) [odds ratio (OR), 0.21; 95\% CI, 0.13-0.33; P<0.00001]. The OR for myocardial infarction (MI) (OR, 0.46; 95\% CI, 0.15-1.47; P=0.19) did not reach statistical significance. PCBs were associated with similar outcomes when compared with DESs with regard to LLL (WMD, -0.04; 95\% CI, -0.18-0.10; P=0.57), MACEs (OR, 0.74; 95\% CI, 0.36-1.53; $\mathrm{P}=0.42$ ) and the ORs for all endpoints, including total mortality, target lesion revascularization, MI, stent thrombosis and binary restenosis, and no statistically significant differences were found. This meta-analysis showed that PCBs are associated with superior outcomes when compared with UCBs in the management of ISR, and are at least as efficacious and as well tolerated as DESs.
\end{abstract}

Correspondence to: Professor Xiao-Yan Li, Department of Cardiology, Renmin Hospital of Wuhan University, 99 Ziyang Road, Wuhan, Hubei 430060, P.R. China

E-mail:xy.li@whu.edu.cn

Key words: paclitaxel-coated balloon, drug-eluting stent, in-stent restenosis, meta-analysis

\section{Introduction}

Percutaneous coronary intervention (PCI) with coronary stent implantation is one of the revolutionary advancements made in the treatment of coronary heart diseases over the past decades. Bare metal stents (BMSs) and drug-eluting stents (DESs) are the two main types of stents used for coronary lesions. Although the incidence of in-stent restenosis (ISR) following stent implantation has been reduced to $<10 \%$ following the transition from BMSs to DESs, its occurrence is not negligible due to the large number of patients treated with BMSs or DESs, particularly patients at high-risk of ISR $(1,2)$; therefore, the optimal treatment for ISR remains undefined. Several randomized trials that compared DES use to conventional therapy for restenosis indicated that DESs exhibit superior clinical and angiographic results (3-5); however, the treatment of ISR with a new type of DES is strongly dependent on long-term dual-antiplatelet therapy, which could prove troublesome for patients who are at high risk of bleeding (6). Furthermore, DESs reduce the flexibility of the vessel and limit the repeatability of the procedure.

Paclitaxel-coated balloon (PCB) technology has emerged as a potential therapeutic alternative in PCI. The PCB is a semi-compliant angioplasty balloon, covered with an antirestenotic drug that is rapidly released locally into the vessel wall as the balloon is inflated (7). The significant advantages of this device include the homogeneous transfer of the drug to the entire vessel wall and the absence of polymer, which reduces the chance of late thrombosis (7). A number of registry data support the safety and feasibility of the use of PCBs in ISR. In particular, the use of PCBs in the treatment of BMS-ISR has achieved notable outcomes (8); however, the majority of randomized clinical trials are limited due to small sample sizes and different follow-up durations. Furthermore, compared with the widespread successful application of DESs, it is uncertain whether additional benefits could be provided by PCBs. The present meta-analysis was therefore conducted with the objective of systematically reviewing the current randomized evidence regarding the clinical and angiographic outcomes of PCBs for patients with ISR.

\section{Materials and methods}

Identification of studies. The MEDLINE, Embase and Cochrane Central Register of Controlled Trials databases were searched 
in April 2014 in order to identify eligible clinical controlled trials comparing PCBs with uncoated balloons (UCBs) or DESs in patients with ISR (BMS-ISR or DES-ISR). No restrictions were imposed on the patients, sample size, language, publication status, lesion type or follow-up duration. The keywords included (drug-coated balloon or drug eluting balloon) and (paclitaxel-coated balloon or paclitaxel-eluting balloon), for the theme 'clinical trial'. Relevant reviews and reference lists of retrieved records were also screened. Two authors selected the studies independently, and disagreements were resolved by discussion.

Selection criteria. All published and/or ongoing clinical controlled trials that focused on the comparison between PCBs and UCBs/DESs for ISR lesions were considered eligible. The clinical and angiographic outcomes were followed up for $\geq 6$ months. Duplicate studies and studies that failed to make adjustments for potential confounders or to provide sufficient statistical analysis were excluded.

Clinical outcomes and definitions. Data for baseline variables and clinical and angiographic outcomes were collected. Outcomes of interest included total mortality (TM), myocardial infarction (MI; defined according to each study protocol), major adverse cardiac events (MACEs), target lesion revascularization (TLR; defined as re-intervention on the index treated lesion), stent thrombosis [ST; defined by the Academic Research Consortium as 'definite' or 'probable' ST (9)], binary restenosis (BR; defined as $\geq 50 \%$ luminal diameter stenosis by quantitative coronary angiography) and in-lesion late lumen loss (LLL). LLL was defined as the difference between the postprocedural and follow-up in-stent or in-segment minimal lumen diameter, as evaluated by quantitative coronary angiography.

Statistical analysis. Dichotomous data were calculated as odds ratios (ORs) and 95\% confidence intervals (95\% CI). Continuous variables are presented as weighted mean difference (WMD) with a 95\% CI. P $<0.05$ was considered to indicate a statistically significant difference. In the presence of inter-study heterogeneity a random-effects model was used, whereas a fixed-effects model was applied in the absence of heterogeneity. Following data pooling, statistical heterogeneity was identified and evaluated by means of the $\mathrm{I}^{2}$ statistic, which represents the proportion of the total variability across studies ( $\mathrm{I}^{2}<25 \%$, trivial heterogeneity; $\mathrm{I}^{2}<50 \%$, moderate heterogeneity; $I^{2}>50 \%$, substantial and important heterogeneity). Analysis was performed using Review Manager Software version 5.1 (The Nordic Cochrane Collaboration, Copenhagen, Denmark).

\section{Results}

Characteristics of the studies. Results from the literature search are depicted in Fig. 1. In total, 171 articles were reviewed, and 9 studies (10-18) [8 randomized controlled trials (RCTs) (10-17) and 1 non-RCT) (18)] with a total of 1,488 patients $(1,608$ lesions) met the criteria for inclusion in the present meta-analysis. Among those patients, PCBs were used in 733 patients (779 lesions), while the control

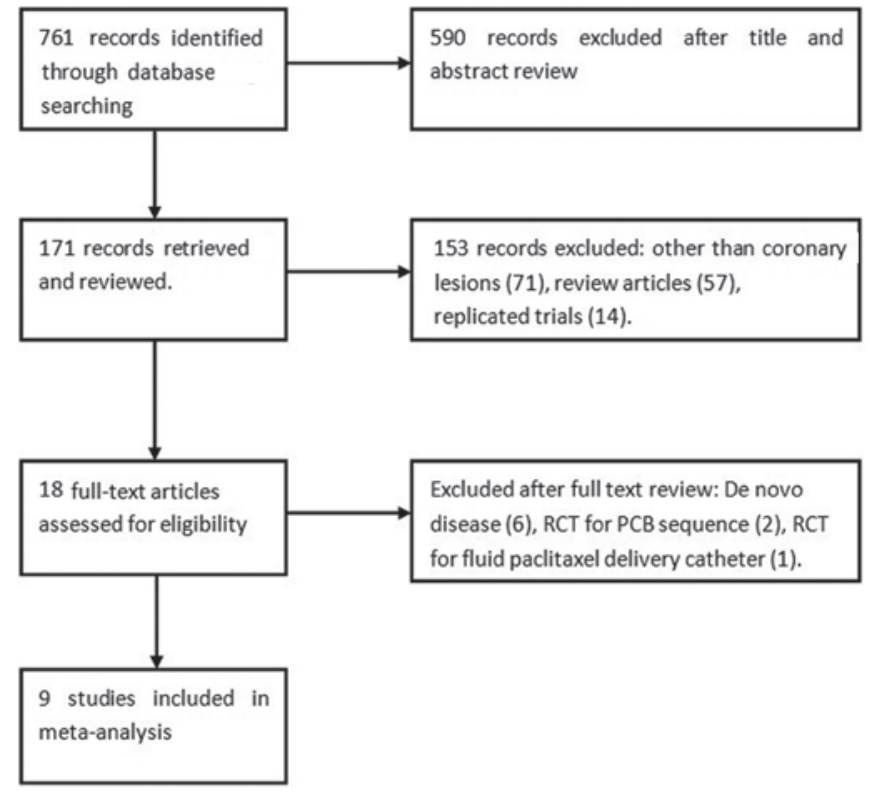

Figure 1. Flow chart of the selection of studies. RCT, randomized controlled trial; PCB, paclitaxel-coated balloon.

treatments included UCBs (317 patients, 348 lesions) and DESs (438 patients, 481 lesions). One of the studies, the ISAR-DESIRE 3 trial (15), was a three-arm trial comparing $\mathrm{PCB}, \mathrm{UCB}$ and DES groups; this study was therefore treated as 2 separate trials. Ultimately, 5 studies comparing PCBs with UCBs and 5 comparing PCBs with DESs were selected. All 9 trials had a clinical primary endpoint, with a follow-up duration ranging from 6 to 9 months, and 8 studies had an angiographic primary endpoint, with a follow-up duration ranging from 6 to 12 months. The studies were multi-center in 7 cases and single center in 2. Among the 8 RCTs, 1 study was double-blind, 3 were single-blind and 4 were unblinded. The demographic and clinical characteristics of the patients are summarized in Tables I and II. No significant difference was found between the PCB and control groups in the baseline characteristics of the patients.

\section{Angiographic outcomes}

LLL. At a follow-up period of 6-9 months, data on LLL were recorded in 5 PCB versus $\mathrm{UCB}$ studies and $4 \mathrm{PCB}$ versus DES studies. A statistical heterogeneity was observed in subgroup PCB versus DES $\left(\mathrm{I}^{2}=66 \% ; \mathrm{P}=0.03\right)$; therefore the random-effects model was used. Comparable results were found in the two subgroups. Patients treated with PCBs exhibited a significant reduction in LLL compared with patients treated with UCBs (WMD, -0.46; 95\% CI, (-0.59)-(-0.34); $\mathrm{P}<0.00001)$. The PCB versus DES subgroup analysis showed a similar trend towards lower LLL in the PCB group, although the difference was not significant (WMD, $-0.04 ; 95 \% \mathrm{CI}$, $-0.18-0.10 ; \mathrm{P}=0.57$ ) (Fig. 2).

$B R$. At a follow-up period of 6-9 months, the rate of $\mathrm{BR}$ recorded in the 5 PCB versus UCB and 4 PCB versus DES studies was analyzed by the random-effects model, since a significant study heterogeneity was observed $\left(\mathrm{I}^{2}=52 \% ; \mathrm{P}=0.08\right)$ in subgroup PCB versus UCB. A statistically significant effect favoring PCB was detected in the PCB versus UCB subgroup 
analysis (OR, 0.13 ; 95\% CI, 0.07-0.25; $\mathrm{P}<0.00001)$. PCB versus DES subgroup analysis showed a benefit associated with DES use, although the difference between PCB and DES use was not significant (OR, 0.95; 95\% CI, 0.56-1.61; $\mathrm{P}=0.84$ ) (Fig. 3).

\section{Clinical outcomes}

$T L R$. At a follow-up period of 6-12 months, TLR data were acquired from all 9 studies. Statistical heterogeneity was noted in the two subgroups $\left(\mathrm{I}^{2}=68 \%\right.$; $\mathrm{P}=0.02$ for $\mathrm{PCB}$ versus $\mathrm{UCB}$ and $\mathrm{I}^{2}=64 \% ; \mathrm{P}=0.03$ for $\mathrm{PCB}$ versus $\mathrm{DES}$ ) and therefore the random-effects model was used. Five out of the 9 studies were included in the PCB versus UCB subgroup analysis. The risk of TLR was significantly lower in patients treated with PCBs (OR, 0.16; 95\% CI, 0.07-0.36; P<0.00001). No significant difference was found in the incidence rate of TLR between the PCB and DES groups (OR, 1.04; 95\% CI, 0.47-2.33; $\mathrm{P}=0.92$ ) (Fig. 4).

MI. At a follow-up period of 6-12 months, MI data were acquired from all 9 studies. A fixed-effect model was selected and the test for heterogeneity showed that the subgroup differences were all non-significant $\left(\mathrm{I}^{2}=21 \% ; \mathrm{P}=0.28\right.$ for $\mathrm{PCB}$ versus $\mathrm{UCB}$ and $\mathrm{I}^{2}=0 \% ; \mathrm{P}=0.97$ for $\mathrm{PCB}$ versus DES). No significant difference between the effect of PCBs and UCBs on the incidence of MI was identified in the subgroup analysis (OR, 0.46; 95\% CI, 0.15-1.47; P=0.19). Similarly, the incidence rate of MI was comparable following PCB and DES implantation (OR, 0.64; 95\% CI, 0.30-1.36; P=0.24) (Fig. 5).

MACEs. At a follow-up period of 6-12 months, MACE results were obtained from 8 out of the 9 studies. Heterogeneity was observed in subgroup PCB versus DES $\left(\mathrm{I}^{2}=57 \% ; \mathrm{P}=0.07\right)$ and therefore a random-effects model was selected. Subgroup analysis showed that PCB treatment had an advantage over UCB treatment in reducing the incidence of MACEs (OR, 0.21 ; 95\% CI, 0.13-0.33; P<0.00001). Furthermore, PCB treatment appeared to be equally efficacious to DES treatment in reducing the incidence rate of MACEs (OR, 0.74; 95\% CI, 0.36-1.53; $\mathrm{P}=0.42$ ) (Fig. 6).

ST. At a follow-up period of 6-12 months, ST results were obtained from 8 out of the 9 studies. Heterogeneity was observed in the PCB versus UCB subgroup analysis $\left(\mathrm{I}^{2}=61 \%\right.$; $\mathrm{P}=0.11)$ and therefore a random-effects model was used. The PCB versus UCB subgroup analysis showed that the two treatments had a similar effect in reducing the incidence of ST (OR, 0.47; 95\% CI, 0.02-10.33; P=0.63), and similar results were also found in the PCB versus DES subgroup analysis (OR, 1.00; 95\% CI, 0.23-4.43; P>0.99) (Fig. 7)

$T M$. At a follow-up period of 6-12 months, TM data were acquired from all 9 studies. The incidence of TM was significantly lower in the PCB-treated patients than in the UCB-treated patients (OR, 0.32; 95\% CI, 0.12-0.83; $\mathrm{P}=0.02)$. No significant difference was detected in the incidence of TM between the PCB and DES groups (OR, 0.91; 95\% CI, 0.41-2.00; $\mathrm{P}=0.81)$. A fixed-effect model was selected due to the absence of heterogeneity in the two subgroups $\left(\mathrm{I}^{2}=0 \%\right.$, $\mathrm{P}=0.39$ for $\mathrm{PCB}$ versus $\mathrm{UCB} ; \mathrm{I}^{2}=21 \%, \mathrm{P}=0.28$ for $\mathrm{PCB}$ versus DES) (Fig. 8).

Sensitivity analysis. Since the test for heterogeneity showed significant differences in the LLL, BR, TLR, MACE and TM results between the PCB and control groups, subgroup analysis was performed according to the different control groups, 
Table II. Baseline characteristics of the patients.

\begin{tabular}{|c|c|c|c|c|c|c|c|c|}
\hline \multirow[b]{2}{*}{ Study name/first author (ref.) } & \multirow[b]{2}{*}{ Group } & \multirow[b]{2}{*}{$\begin{array}{l}\text { No. of } \\
\text { patients }\end{array}$} & \multirow[b]{2}{*}{$\begin{array}{l}\text { Mean age } \\
\text { (years) }\end{array}$} & \multirow[b]{2}{*}{$\begin{array}{l}\mathrm{DM} \\
(\%)\end{array}$} & \multicolumn{2}{|c|}{ Before procedure (mm) } & \multicolumn{2}{|c|}{ Follow-up (n) } \\
\hline & & & & & MLD & $\begin{array}{l}\text { Lesion } \\
\text { length }\end{array}$ & Angiographic & Clinical \\
\hline \multirow[t]{2}{*}{ PACCOCATH ISR I/II (10) } & PCB & 54 & $65.4 \pm 10.3$ & 17 & $0.63 \pm 0.29$ & $18.3 \pm 9.7$ & 48 & 54 \\
\hline & UCB & 54 & $66.3 \pm 9.8$ & 41 & $0.70 \pm 0.35$ & $18.6 \pm 8.3$ & 49 & 54 \\
\hline \multirow[t]{2}{*}{ PEPCAD-DES (9) } & PCB & 72 & $69.8 \pm 10.8$ & 36.1 & $0.66 \pm 0.40$ & $11.2 \pm 6.5$ & 64 & 72 \\
\hline & $\mathrm{UCB}$ & 38 & $64.0 \pm 11.3$ & 34.2 & $0.62 \pm 0.44$ & $12.2 \pm 8.2$ & 31 & 38 \\
\hline \multirow[t]{2}{*}{ Habara (11) } & PCB & 25 & $69.9 \pm 11.0$ & 56 & $0.99 \pm 0.32$ & $12.7 \pm 5.3$ & 23 & 25 \\
\hline & UCB & 25 & $68.9 \pm 9.9$ & 68 & $0.92 \pm 0.51$ & $13.2 \pm 5.5$ & 24 & 25 \\
\hline \multirow[t]{2}{*}{ PEPCAD China ISR (12) } & PCB & 109 & $61.8 \pm 9.3$ & 40.4 & $0.85 \pm 0.38$ & $12.52 \pm 6.55$ & $97^{\mathrm{a}}$ & 109 \\
\hline & PES & 106 & $62.1 \pm 9.3$ & 33.0 & $0.86 \pm 0.41$ & $13.08 \pm 7.13$ & $84^{\mathrm{a}}$ & 106 \\
\hline \multirow[t]{2}{*}{ Unverdorben (13) } & PCB & 66 & $64.6 \pm 9.7$ & 33.3 & $0.74 \pm 0.27$ & $15.7 \pm 6.6$ & 57 & 66 \\
\hline & PES & 65 & $65.1 \pm 8.7$ & 26.2 & $0.77 \pm 0.30$ & $15.4 \pm 6.6$ & 59 & 65 \\
\hline \multirow[t]{3}{*}{ ISAR-DESIRE 3 (14) } & PCB & 137 & $67.7 \pm 10.4$ & 41 & $0.97 \pm 0.48$ & N/A & $147^{\mathrm{a}}$ & 136 \\
\hline & PES & 131 & $68.8 \pm 10.0$ & 47 & $0.93 \pm 0.50$ & N/A & $142^{\mathrm{a}}$ & 127 \\
\hline & UCB & 134 & $67.1 \pm 9.3$ & 37 & $0.88 \pm 0.49$ & N/A & $127^{\mathrm{a}}$ & 129 \\
\hline \multirow[t]{2}{*}{ Habara (15) } & $\mathrm{PCB}$ & 137 & $68.3 \pm 10.3$ & 46 & $0.86 \pm 0.32$ & $12.8 \pm 6.5$ & $139^{\mathrm{a}}$ & 136 \\
\hline & UCB & 71 & $70.4 \pm 10.2$ & 42 & $0.84 \pm 0.34$ & $13.7 \pm 5.8$ & $69^{a}$ & 71 \\
\hline \multirow[t]{2}{*}{ RIBS V (16) } & PCB & 95 & $67 \pm 11$ & 32 & $1.02 \pm 0.4$ & $13.7 \pm 7$ & 84 & 95 \\
\hline & EES & 94 & $64 \pm 12$ & 20 & $0.93 \pm 0.4$ & $13.8 \pm 6$ & 86 & 94 \\
\hline \multirow[t]{2}{*}{ Almalla (17) } & PCB & 46 & $69.6 \pm 9.6$ & 39.1 & $0.57 \pm 0.30$ & $9.0 \pm 5.2$ & N/A & 46 \\
\hline & EES & 40 & $67.7 \pm 10.8$ & 35 & $0.51 \pm 0.41$ & $12.3 \pm 11.0$ & N/A & 40 \\
\hline
\end{tabular}

${ }^{a}$ Number of lesions. DM, diabetes mellitus; EES, everolimus-eluting stent; MLD, minimum lumen diameter; N/A, not available; PCB, paclitaxel-coated balloon; PES, paclitaxel-eluting stent; UCB, uncoated balloon.

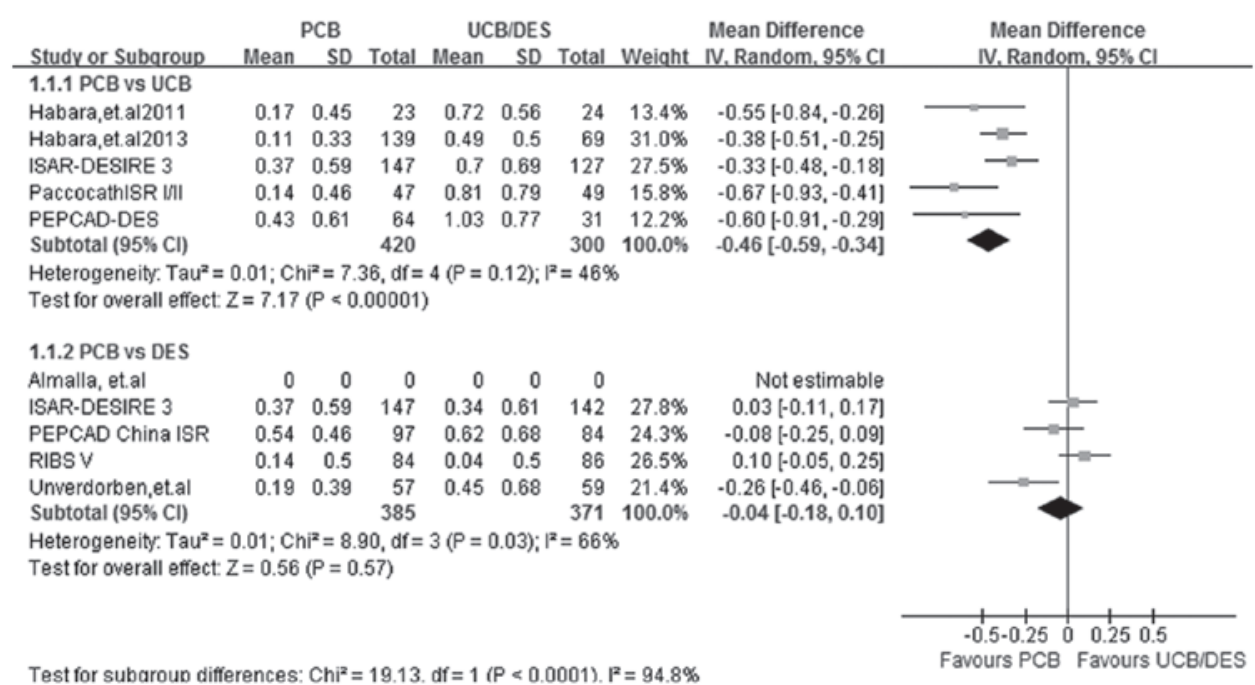

Figure 2. Forest plot of late lumen loss in in-stent restenosis for PCB versus control treatment. UCB, uncoated balloon; DES, drug-eluting stent; OR, odds ratio; $\mathrm{CI}$, confidence interval; PCB, paclitaxel-coated balloon; SD, standard deviation; df, degrees of freedom.

giving $\mathrm{PCB}$ versus $\mathrm{UCB}$ and $\mathrm{PCB}$ versus DES subgroups. By removing one study at a time, a sensitivity analysis was performed. This analysis did not detect any effect of any single study on the results, with the exception of the TM results in the PCB versus UCB subgroup analysis, which indicated that these results were statistically reliable; however, following the removal of the PEPCAD-DES study (10) from the PCB versus UCB subgroup analysis, the significant difference was lost.
We therefore hypothesized that this may have been due to the small sample size of the study and the heterogeneity across the studies included in the subgroup.

\section{Discussion}

Even in the DES era, the treatment of ISR remains a challenge for interventional cardiology. Currently, the placement 


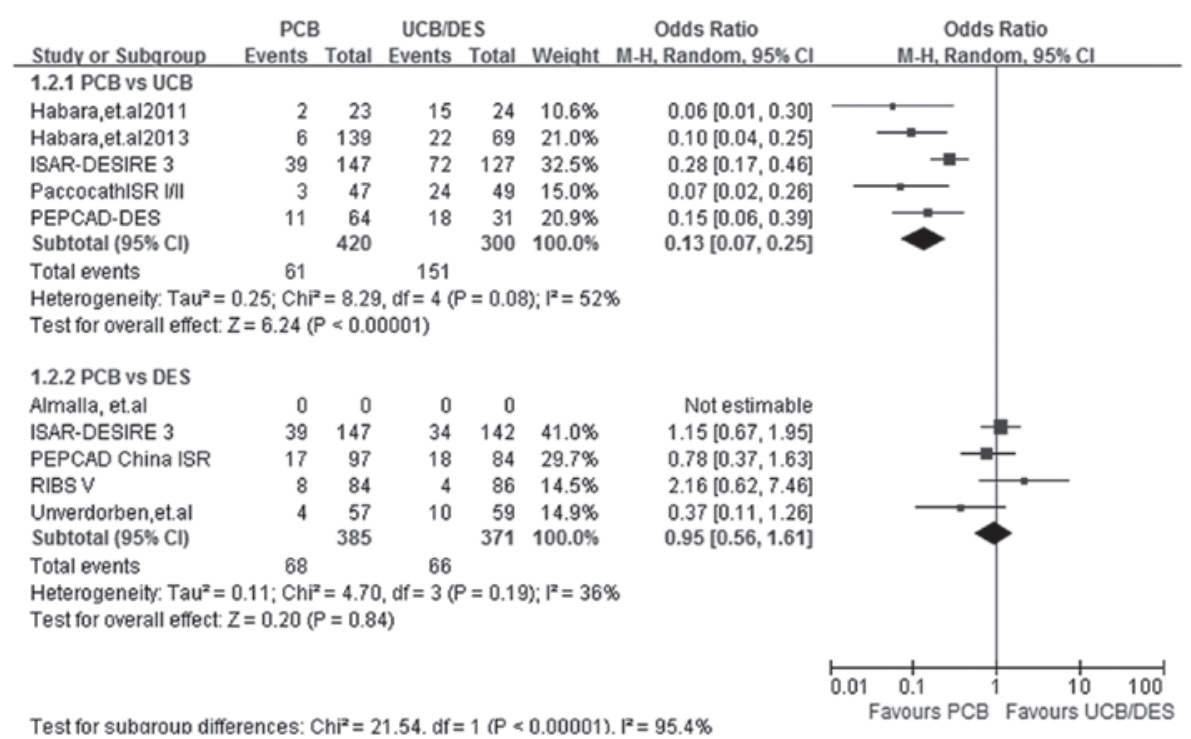

Figure 3. Forest plot of binary restenosis in in-stent restenosis for PCB versus control treatment. UCB, uncoated balloon; DES, drug-eluting stent; OR, odds ratio; CI, confidence interval; $\mathrm{PCB}$, paclitaxel-coated balloon; df, degrees of freedom.

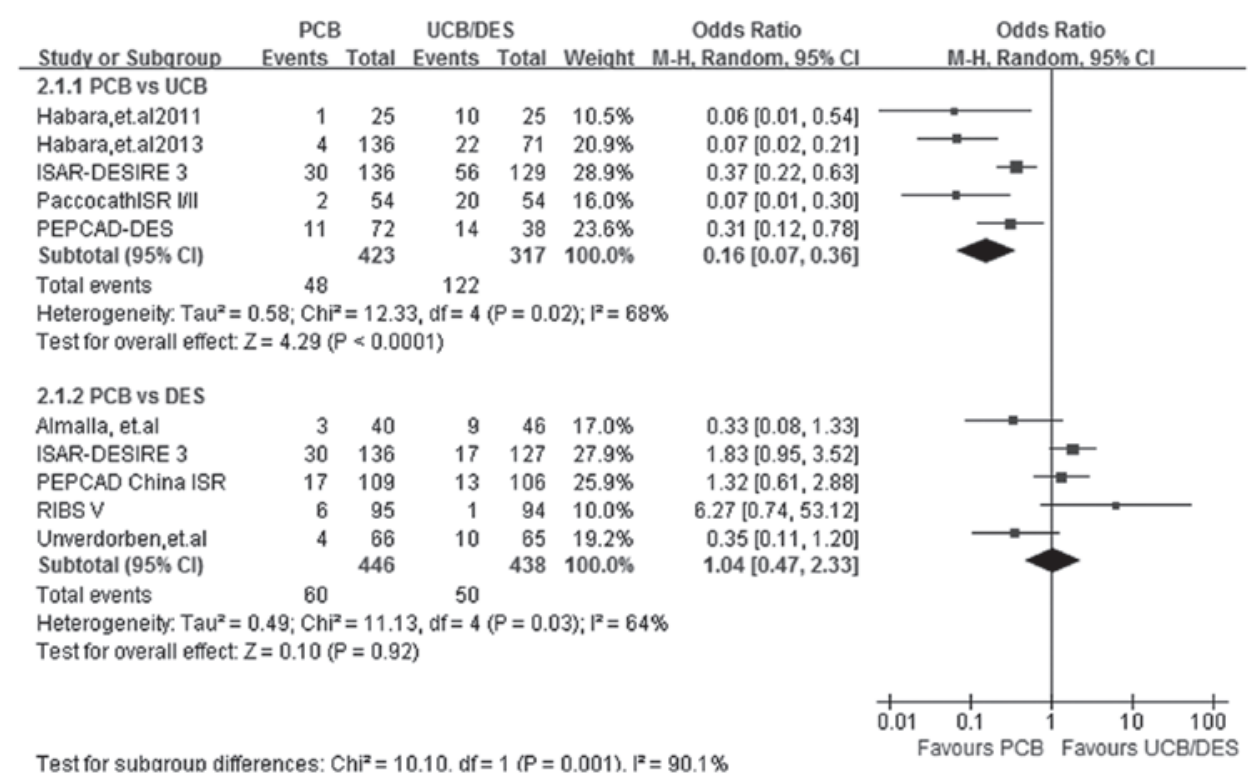

Figure 4. Forest plot of target lesion revascularization in in-stent restenosis for PCB versus control treatment. UCB, uncoated balloon; DES, drug-eluting stent; OR, odds ratio; CI, confidence interval; PCB, paclitaxel-coated balloon; df, degrees of freedom.

of a second (drug-eluting) stent appears to be the most effective treatment method (19); however, it is still controversial whether or not DES implantation could have a positive outcome in high-risk patients with diabetes mellitus or minor or diffuse vessel lesion. This controversy prompted the development of PCB devices as a therapeutic alternative for the constantly increasing number of patients suffering from ISR. The first-in-human study to explore PCB use in patients with ISR was the PACCOCATH-ISR trial (20), which demonstrated the superiority of the angiographic and clinical outcomes of PCB treatment as compared with those of UCB treatment after a 6- to 12-month follow-up. Several small-scale studies were subsequently conducted, designed as randomized or non-randomized, single or multicenter, blind or unblinded, and compared with different control groups. None of those studies, however, came to a final conclusion regarding the real effect of PCB in the treatment of ISR. A previous meta-analysis (21) showed that the results of PCB were superior to those of a normal UCB in the treatment of coronary artery disease, and another meta-analysis (22) had similar results when comparing PCB use with traditional treatment methods, including UCBs and DESs. Despite these results, however, both analyses had major limitations: The former meta-analysis did not divide the patients with ISR or de novo lesions into different subgroups and the latter did not divide the control treatments into different subgroups (UCB or DES).

In the present meta-analysis, the pooled results comparing PCB implantation versus UCB and DES implantation in patients with ISR were reported. The principal advantage of this meta-analysis is that it divided 9 clinical 


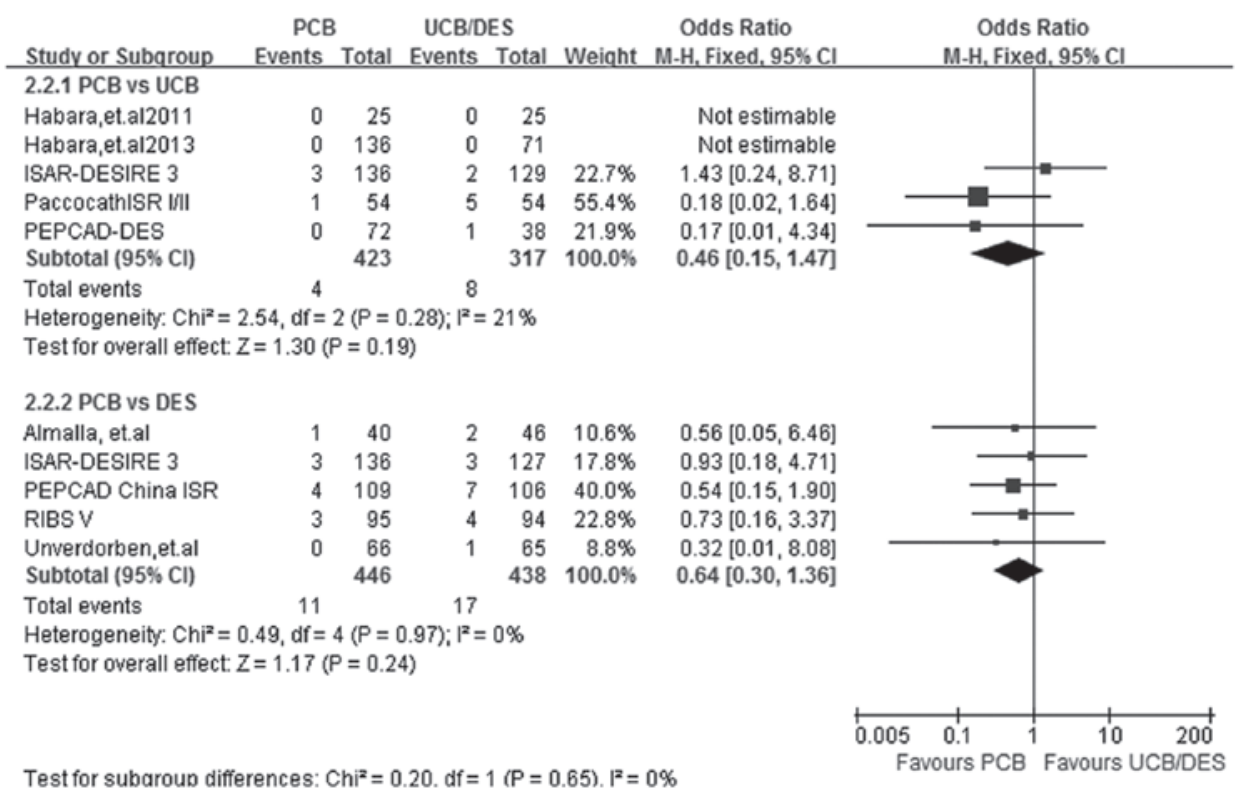

Figure 5. Forest plot of myocardial infarction in in-stent restenosis for PCB versus control treatment. UCB, uncoated balloon; DES, drug-eluting stent; OR, odds ratio; CI, confidence interval; PCB, paclitaxel-coated balloon; df, degrees of freedom.

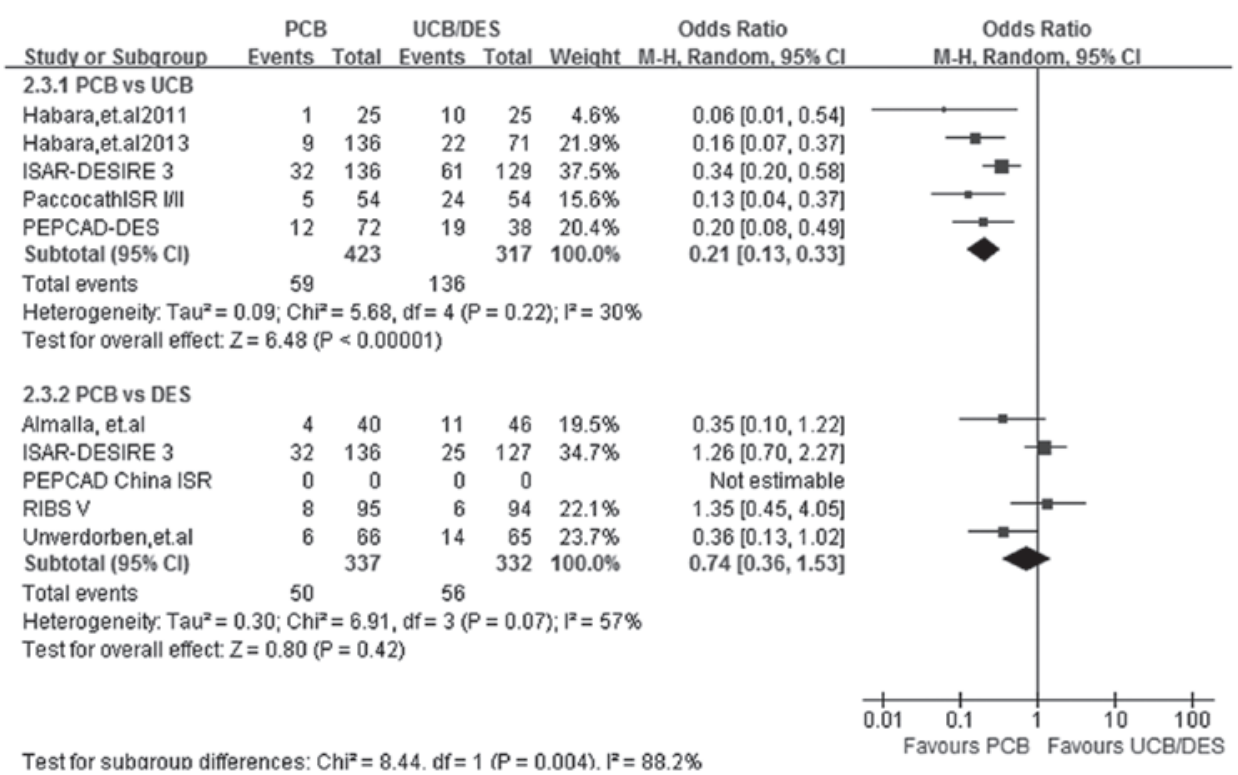

Figure 6. Forest plot of major adverse cardiac events in in-stent restenosis for PCB versus control treatment. UCB, uncoated balloon; DES, drug-eluting stent; $\mathrm{OR}$, odds ratio; CI, confidence interval; PCB, paclitaxel-coated balloon; df, degrees of freedom.

studies (1488 patients, 1,608 lesions) into two subgroups (PCB versus UCB and PCB versus DES). In the PCB versus UCB subgroup analysis, the results of the 6- to 9-month angiographic follow-up showed that PCB treatment had a significant advantage over UCB treatment in reducing the incidence rate of LLL and BR in ISR therapy. In addition, the pooled estimates demonstrated a clear superiority of PCB in the 6- to 12-month follow-up clinical results (TLR, MACEs and TM), and PCBs proved more effective than UCBs in reducing the incidence of MI (4/423 vs. 8/317) and ST (2/369 vs. 4/263); however the difference was non-significant. We hypothesized that this lack of significance may have been due to the small sample size and the lack of ST data in the PACCOCATH-ISR I/II trial (11). In the other subgroup,
5 trials (4 RCTs and 1 NRCT) that compared PCBs and DESs in the treatment of ISR were included. Notably, the pooled data showed that PCBs appeared to be equally effective as DESs in the treatment of ISR, based on angiographic and clinical findings, although moderate to substantial statistical heterogeneity was observed across the studies. We conjectured that the heterogeneity may have occurred due to the fact that the 1 NRCT [Almalla, et al (18)], which was designed as an observational trial and only reported the clinical outcomes at the follow-up period of 12 months, was included in the study. The NRCT was subsequently omitted for further analysis; however, no significant differences were observed in the clinical outcomes (TLR, MI, MACE, ST and TM) between the PCB and DES treatment groups. This clinical evidence 


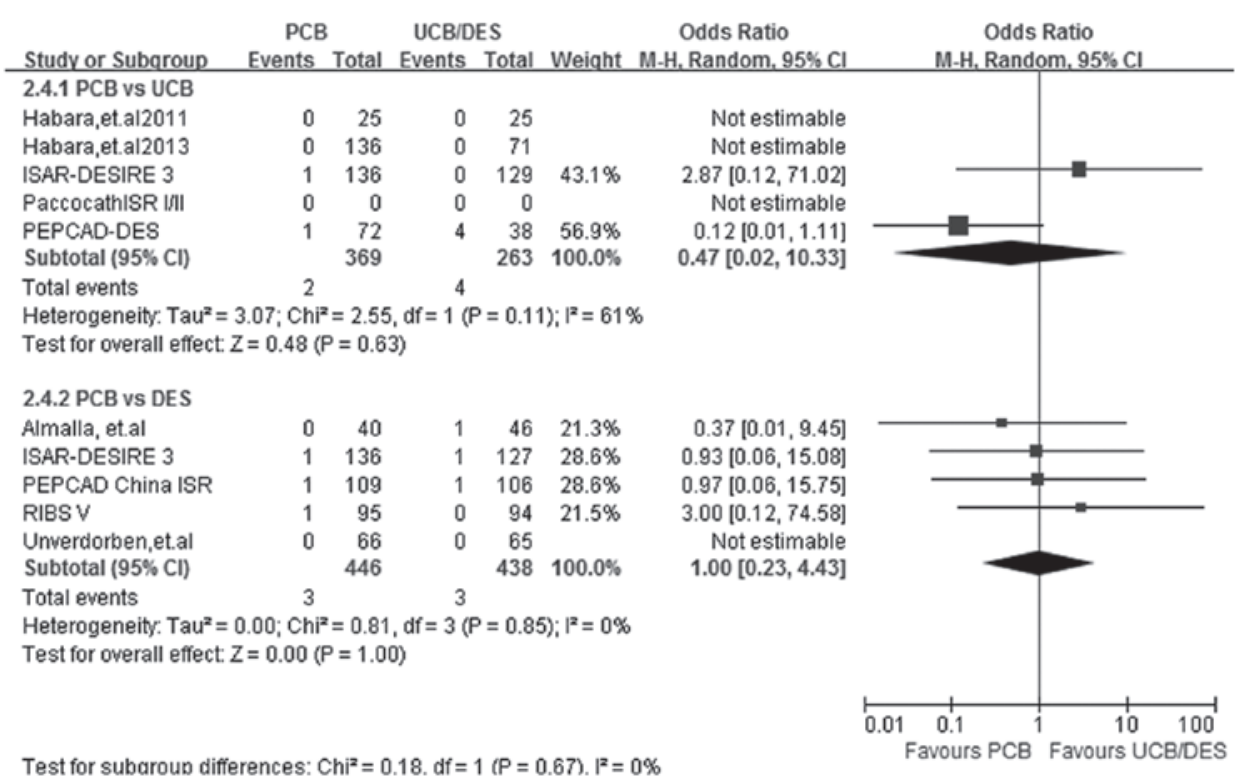

Figure 7. Forest plot of stent thrombosis in in-stent restenosis for PCBversus control treatment. UCB, uncoated balloon; DES, drug-eluting stent; OR, odds ratio; $\mathrm{CI}$, confidence interval; $\mathrm{PCB}$, paclitaxel-coated balloon; df, degrees of freedom.

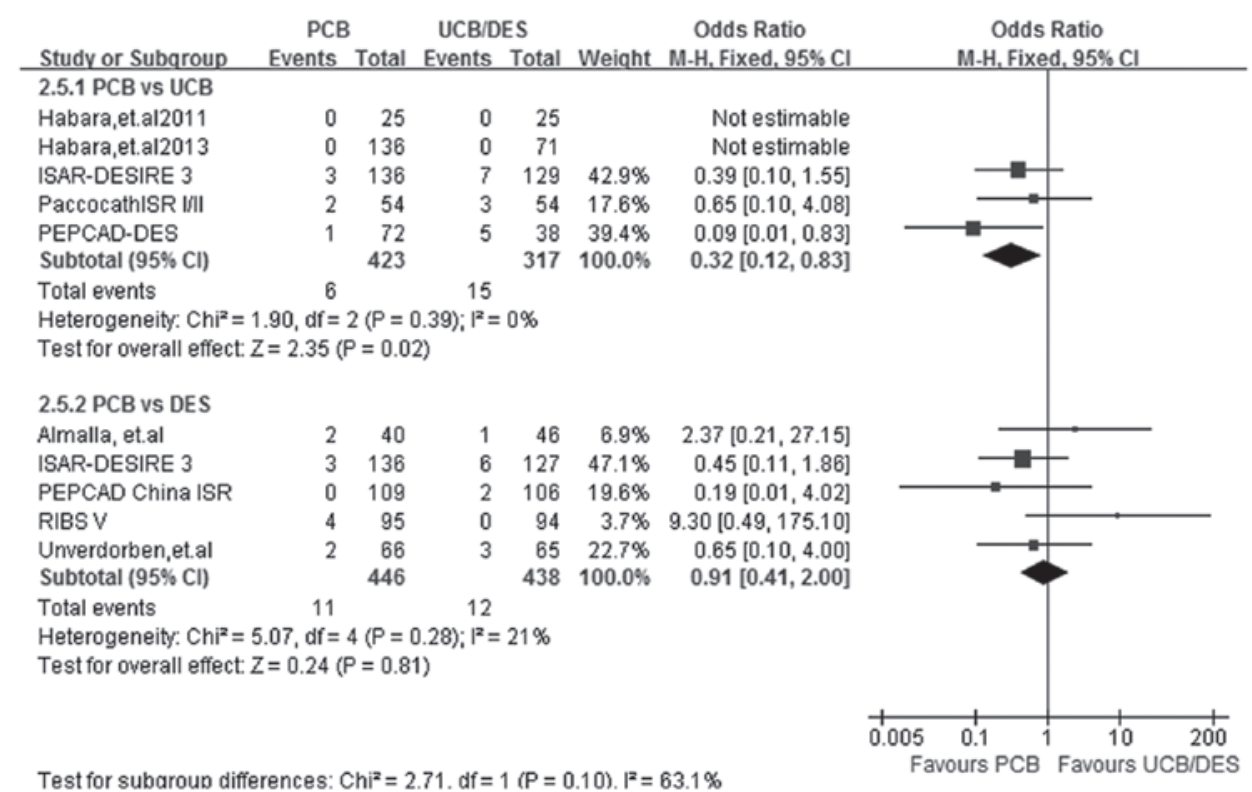

Figure 8. Forest plot of total mortality in in-stent restenosis for PCB versus control treatment. UCB, uncoated balloon; DES, drug-eluting stent; OR, odds ratio; CI, confidence interval; PCB, paclitaxel-coated balloon; df, degrees of freedom.

supported the hypothesis that PCBs are comparable to DESs in the treatment of coronary ISR with regard to efficacy and safety, obviating the necessity of implanting an additional metal layer.

Previous studies have indicated that there are significant differences between the occurrence of ISR following BMS and DES implantion $(23,24)$. These differences manifest themselves in various ways, such as time of presentation, morphological patterns, underlying mechanisms, tissue composition and response to implantation (25). The angiographic pattern of ISR is different following BMS implantation from that subsequent to DES implantation. The angiographic pattern of DES-ISR is predominantly focal and is associated with better prognosis, whereas diffuse or proliferative patterns are rare. On the contrary, a non-focal pattern frequently occurs following BMS implantation and is associated with a high incidence of restenosis (2). It is apparent that head-to-head comparisons between the use of PCBs for the treatment of BMS-ISR and DES-ISR are lacking. The SeQuentPlease World Wide Registry (26) was a large-scale, prospective registry study in which the TLR rate was significantly lower in patients who underwent a PCB angiography for BMS-ISR compared with that in patients who underwent a PCB angiography for DES-ISR (3.8 vs. 9.6\%, $\mathrm{P}<0.001)$. In 5 studies $(10,12,13,15,18)$ in the present meta-analysis, the restenosis type being treated was DES-ISR, and a comparison between PCB and control (UCB or DES) treatments was made. The clinical and angiographic results reported for the $\mathrm{PCB}$ treatment were considered to be 
comparable to those for the control treatments; therefore, we consider PCB to be an attractive optimal treatment strategy for DES and BMS restenosis, despite the effect of PCBs on DES restenosis being shown to be relatively inferior to that on BMS restenosis (26).

Several limitations were noted in this meta-analysis. Firstly, since one of the included studies was an observational registry study, it was more likely to have been biased in terms of selection, performance, attrition and detection. Secondly, the definition of angiographic outcomes (LLL and BR) differed in each study due to the angiographic measurements being performed at the target lesion either over the entire length of the study device (in-stent) or within $5 \mathrm{~mm}$ proximal and distal to the target lesion (in-segment). Thirdly, the short follow-up period (6-12 months) in all studies was inadequate for detecting late adverse events, such as very late thrombosis. In addition, despite the fact that the control groups were split into UCB and DES subgroups and analyzed using a random-effects model or for sensitivity to minimize heterogeneity, a potential heterogeneity in the methods, patients, sample size and baseline characteristics of the patients still existed; therefore caution should be taken when interpreting the results.

In conclusion, this meta-analysis, based on the currently available angiographic and clinical data from clinical evidence, shows a significant superiority of PCB over UCB in the treatment of ISR. Furthermore, the findings support the conclusion that PCB implantation is at least as effective and tolerable as DES implantation. These results cannot, however, replace the results of those multicenter, prospective RCTs with a long follow-up duration in critically evaluating the more reliable 'real-world' clinical evidence found in PCB-treated patients with ISR.

\section{References}

1. Moses JW, Leon MB, Popma JJ, et al; SIRIUS Investigators: Sirolimus-eluting stents versus standard stents in patients with stenosis in a native coronary artery. N Engl J Med 349: 1315-1323, 2003.

2. Dangas GD, Claessen BE, Caixeta A, Sanidas EA, Mintz GS and Mehran R: In-stent restenosis in the drug-eluting stent era. J Am Coll Cardiol 56: 1897-1907, 2010.

3. Kim YH, Lee BK, Park DW, et al: Comparison with conventional therapies of repeated sirolimus-eluting stent implantation for the treatment of drug-eluting coronary stent restenosis. Am J Cardiol 98: 1451-1454, 2006.

4. Mishkel GJ, Moore AL, Markwell S, Shelton MC and Shelton ME: Long-term outcomes after management of restenosis or thrombosis of drug-eluting stents. J Am Coll Cardiol 49: 181-184, 2007.

5. Stone GW, Ellis SG, O'Shaughnessy CD, et al; TAXUS V ISR Investigators: Paclitaxel-eluting stents vs vascular brachytherapy for in-stent restenosis within bare-metal stents: The TAXUS V ISR randomized trial. JAMA 295: 1253-1263, 2006.

6. Bhatt DL: Intensifying platelet inhibition - navigating between Scylla and Charybdis. N Engl J Med 357: 2078-2081, 2007.

7. Loh JP and Waksman R: Paclitaxel drug-coated balloons: A review of current status and emerging applications in native coronary artery de novo lesions. JACC Cardiovasc Interv 5: 1001-1012, 2012.

8. Gray WA and Granada JF: Drug-coated balloons for the prevention of vascular restenosis. Circulation 121: 2672-2680, 2010 .
9. Cutlip DE, Windecker S, Mehran R, et al; Academic Research Consortium: Clinical end points in coronary stent trials: A case for standardized definitions. Circulation 115: 2344-2351, 2007.

10. Rittger H, Brachmann J, Sinha AM, et al: A randomized, multicenter, single-blinded trial comparing paclitaxel-coated balloon angioplasty with plain balloon angioplasty in drug-eluting stent restenosis: The PEPCAD-DES study. J Am Coll Cardiol 59: 1377-1382, 2012.

11. Scheller B, Hehrlein C, Bocksch W, et al: Two year follow-up after treatment of coronary in-stent restenosis with a paclitaxel-coated balloon catheter. Clin Res Cardiol 97: 773-781, 2008.

12. Habara S, Mitsudo K, Kadota K, et al: Effectiveness of paclitaxel-eluting balloon catheter in patients with sirolimus-eluting stent restenosis. JACC Cardiovasc Interv 4: 149-154, 2011.

13. Xu B, Gao R, Wang J, et al; PEPCAD China ISR Trial Investigators: A prospective, multicenter, randomized trial of paclitaxel-coated balloon versus paclitaxel-eluting stent for the treatment of drug-eluting stent in-stent restenosis: Results from the PEPCAD China ISR trial. JACC Cardiovasc Interv 7: 204-211, 2014.

14. Unverdorben M, Vallbracht C, Cremers B, et al: Paclitaxel-coated balloon catheter versus paclitaxel-coated stent for the treatment of coronary in-stent restenosis. Circulation 119: 2986-2994, 2009.

15. Byrne RA, Neumann FJ, Mehilli J, et al; ISAR-DESIRE 3 investigators: Paclitaxel-eluting balloons, paclitaxel-eluting stents, and balloon angioplasty in patients with restenosis after implantation of a drug-eluting stent (ISAR-DESIRE 3): A randomised, open-label trial. Lancet 381: 461-467, 2013.

16. Habara S, Iwabuchi M, Inoue N, et al: A multicenter randomized comparison of paclitaxel-coated balloon catheter with conventional balloon angioplasty in patients with bare-metal stent restenosis and drug-eluting stent restenosis. Am Heart J 166: 527-533, 2013.

17. Alfonso F, Pérez-Vizcayno MJ, Cárdenas A, et al; RIBS V Study Investigators, under the auspices of the Working Group on Interventional Cardiology of the Spanish Society of Cardiology: A randomized comparison of drug-eluting balloon versus everolimus-eluting stent in patients with bare-metal stent-in-stent restenosis: The RIBS V Clinical Trial (Restenosis Intra-stent of Bare Metal Stents: Paclitaxel-eluting balloon vs everolimus-eluting stent). J Am Coll Cardiol 63: 1378-1386, 2014.

18. Almalla M, Schröder J, Pross V, Marx N and Hoffmann R: Paclitaxel-eluting balloon versus everolimus-eluting stent for treatment of drug-eluting stent restenosis. Catheter Cardiovasc Interv 83: 881-887, 2014.

19. Kastrati A, Mehilli J, von Beckerath N, et al; ISAR-DESIRE Study Investigators: Sirolimus-eluting stent or paclitaxel-eluting stent vs balloon angioplasty for prevention of recurrences in patients with coronary in-stent restenosis: A randomized controlled trial. JAMA 293: 165-171, 2005.

20. Scheller B, Hehrlein C, Bocksch W, et al: Treatment of coronary in-stent restenosis with a paclitaxel-coated balloon catheter. N Engl J Med 355: 2113-2124, 2006.

21. Yu CM, Kwong JS and Sanderson JE: Drug-eluting balloons for coronary artery disease: A meta-analysis of randomized controlled trials. Int J Cardiol 168: 197-206, 2013.

22. Navarese EP, Austin D, Gurbel PA, et al: Drug-coated balloons in treatment of in-stent restenosis: A meta-analysis of randomised controlled trials. Clin Res Cardiol 102: 279-287, 2013.

23. Steinberg DH, Gaglia MA Jr, Pinto Slottow TL, et al: Outcome differences with the use of drug-eluting stents for the treatment of in-stent restenosis of bare-metal stents versus drug-eluting stents. Am J Cardiol 103: 491-495, 2009.

24. Byrne RA, Cassese S, Windisch T, et al: Differential relative efficacy between drug-eluting stents in patients with bare metal and drug-eluting stent restenosis; evidence in support of drug resistance: Insights from the ISAR-DESIRE and ISAR-DESIRE 2 trials. EuroIntervention 9: 797-802, 2013.

25. Alfonso F: Treatment of drug-eluting stent restenosis the new pilgrimage: Quo vadis? J Am Coll Cardiol 55: 2717-2720, 2010.

26. Wöhrle J, Zadura M, Möbius-Winkler S, et al: SeQuentPlease World Wide Registry: Clinical results of SeQuent please paclitaxel-coated balloon angioplasty in a large-scale, prospective registry study. J Am Coll Cardiol 60: 1733-1738, 2012. 